\title{
Robust Propagation of Probability Boxes by Interval Predictor Models
}

\author{
Jonathan Sadeghi ${ }^{1}$, Marco De Angelis ${ }^{1}$, and Edoardo Patelli ${ }^{1}$ \\ ${ }^{1}$ edoardo.patelli@liverpool.ac.uk, Institute for Risk and \\ Uncertainty, Chadwick Building, University of Liverpool, Peach \\ Street, Liverpool L69 7ZF, United Kingdom
}

August 28, 2019

\begin{abstract}
This paper proposes numerical strategies to robustly and efficiently propagate probability boxes through expensive black box models. An interval is obtained for the system failure probability, with a confidence level. The three proposed algorithms are sampling based, and so can be easily parallelised, and make no assumptions about the functional form of the model. In the first two algorithms, the performance function is modelled as a function with unknown noise structure in the aleatory space and supplemented by a modified performance function. In the third algorithm, an Interval Predictor Model is constructed and a re-weighting strategy used to find bounds on the probability of failure. Numerical examples are presented to show the applicability of the approach. The proposed method is flexible and can account for epistemic uncertainty contained inside the limit state function. This is a feature which, to the best of the authors' knowledge, no existing methods of this type can deal with.
\end{abstract}

\section{Introduction}

In structural reliability analysis, the aim is to compute the probability that the performance of the system is less than some specified threshold; this probability is known as the failure probability of the system. In general, the failure probability can be computed by Monte Carlo simulation. Firstly, the performance of the system is defined as a function of the system variables, $g(\boldsymbol{x})$. The performance function is negative when the system fails, and otherwise positive. Therefore, the Monte Carlo estimator for the failure probability is

$$
P_{f}=\int I_{f}(\boldsymbol{x}) f_{X}(\boldsymbol{x}) d \boldsymbol{x}=\frac{1}{N} \sum_{i=1}^{N} I_{f}\left(\boldsymbol{x}_{i}\right)
$$


This manuscript version is made available under the CC-BY-NC-ND 4.0

where $N$ samples, $\boldsymbol{x}_{i}$, are drawn from the the probability density function of the system random variables $f_{X}(\boldsymbol{x})$, and passed through the indicator function $I_{f}(\boldsymbol{x})$, which is 1 when $g(\boldsymbol{x})<0$ and 0 otherwise.

Probability boxes make possible the modelling of epistemic uncertainties in the parameters of cumulative distribution functions [12]. The probability density function $f_{X}(\boldsymbol{x})$ is no longer known exactly; instead only a set of probability density functions are available. For this reason, probability boxes are useful in situations where limited data is available to model random variables. An example of such a situation in the nuclear industry is given by [22, and in the aerospace industry by [21. However, due to their epistemic uncertainty, the propagation of probability boxes through models is more difficult than for conventional random variables, as in general a naïve Monte Carlo propagation with insufficient samples will discard some of the epistemic uncertainty [8, 9].

To combat this problem, sophisticated optimisation techniques have been used. However, these are often computationally expensive for black box models as the gradient of the objective function may be unknown, or difficult to compute. In addition, for some models there is no theoretical guarantee that the optimisation will converge to the global optimum. This is troubling, because convergence to a point other than the true optimum threatens the robust analysis which probability boxes are designed to provide.

In the literature, some metamodeling techniques have been proposed to reduce the computational demands of the analysis, which would allow the objective function to be evaluated with less computational expense [32, 26, 27, 16]. However, these techniques are usually dependent on the assumptions required to construct the metamodel, which may be implicit or explicit. For example, 8 does not explicitly construct a metamodel, but implicitly the limit state function is modelled as being only moderately non-linear.

In this paper, a novel technique is proposed which uses Interval Predictor Models - a recently developed type of metamodel which make very few assumptions [7, 25] - to propagate distributional probability boxes through black box models. Interval Predictor Models intrinsically quantify uncertainty, and the reliability of this uncertainty quantification can be assessed using recent advancements in scenario optimisation [6] (i.e. optimisation with sampled, randomised constraints). This presents advantages over direct implementation of the double loop Monte Carlo algorithm. In [13, it was acknowledged that optimising a non-smooth function is a challenging aspect of double loop Monte Carlo simulation; by using an IPM, smooth bounds on the model response can be obtained even if the response of the model under analysis is non-continuous.

In the present authors' previous work 24, IPMs were first used to calculate bounds on the failure probability for the probability box propagation problem. The present article advances [24] by introducing new algorithms to yield tighter bounds on the failure probability, comprised of a different sampling strategy and improved techniques for creating IPMs, and providing more extensive numerical experiments. In [11, it is demonstrated that using IPM metamodels for the structural reliability problem with random variables, as opposed to probability boxes, produces bounds on the failure probability which are roughly equivalent 
This manuscript version is made available under the CC-BY-NC-ND 4.0

license http://creativecommons.org/licenses/by-nc-nd/4.0/

○ 2019 .

to those obtained using Gaussian Process metamodels. This paper presents a major generalisation of the results in [11, by considering epistemic uncertainty in probability distribution functions, i.e. probability boxes.

In Section 2, a review of theory relevant to the paper is presented, including Interval Predictor Models and Probability Boxes. In Section 3.1, a novel technique is proposed to propagate distributional probability boxes through black box models, and calculate bounds on the probability of failure, $P_{f}$, of structures in the well known structural reliability analysis problem. This is achieved by modelling the performance function, as a function of the aleatory variables, and then conducting Monte Carlo analysis on the upper and lower bounds. This is effectively a naïve double loop approach using a robust metamodel. In Section 3.2, a modification is proposed to the method in Section 3.1, where the IPM is trained on so-called focal elements in the aleatory space, which are obtained by brute force sampling over the epistemic variables to find the extrema of model response for each point in the aleatory space. In Section 3.3, a similar approach is demonstrated for the general double loop Monte Carlo propagation problem where an IPM is trained on samples of the model from a proposal distribution, and then the samples from the metamodel are re-weighted using importance sampling to find bounds on the probability of failure. Section 4 describes how IPMs used in the literature can be modified to create more accurate metamodels for performance functions. Section 5 demonstrates the application of the developed techniques to the deflection of a cantilever beam with uncertain parameters, a non-linear oscillator, and the modal analysis of a small satellite.

\section{Theoretical Background}

\subsection{Interval Predictor Models}

This section provides a brief outline of the results used in this paper from the theory of sampled constraint optimisation and Interval Predictor Models. Let us consider a black box model (sometimes referred to as the Data Generating Mechanism) which acts on a vector of input variables $\boldsymbol{x} \in \mathbb{R}^{n_{x}}$ to produce an output $y \in \mathbb{R}$. The Data Generating Mechanism will be approximated with an Interval Predictor Model (IPM) which returns an interval for each vector $\boldsymbol{x} \in X$, the set of inputs, given by

$$
I_{y}(\boldsymbol{x}, P)=\{y=G(\boldsymbol{x}, \boldsymbol{p}), \boldsymbol{p} \in P\},
$$

where $G$ is an arbitrary function and $\boldsymbol{p}$ is a parameter vector. In order to be useful, the obtained interval should have the smallest range possible whilst still enclosing all data points generated by the full model. By making an approximation for $G$ and considering a linear parameter dependency Eqn. (2) becomes

$$
I_{y}(\boldsymbol{x}, P)=\left\{y=\boldsymbol{p}^{T} \phi(\boldsymbol{x}), \boldsymbol{p} \in P\right\},
$$


This manuscript version is made available under the CC-BY-NC-ND 4.0

license http://creativecommons.org/licenses/by-nc-nd/4.0/

(1)2019.

where $\phi(\boldsymbol{x})$ is a basis (polynomial and radial bases are commonly used), and $\boldsymbol{p}$ is a member of the hyper-rectangular uncertainty set

$$
P=\{\boldsymbol{p}: \underline{\boldsymbol{p}} \leq \boldsymbol{p} \leq \overline{\boldsymbol{p}}\},
$$

where $\boldsymbol{p}$ and $\overline{\boldsymbol{p}}$ are parameter vectors specifying the defining vertices of the hyper rectangular uncertainty set. The IPM is defined by the interval

$$
I_{y}(\boldsymbol{x}, P)=[\underline{y}(\boldsymbol{x}, \overline{\boldsymbol{p}}, \underline{\boldsymbol{p}}), \bar{y}(\boldsymbol{x}, \overline{\boldsymbol{p}}, \underline{\boldsymbol{p}})],
$$

where $y$ and $\bar{y}$ are the lower and upper bounds of the IPM, respectively. Explicitly, the lower bound is given by

$$
\underline{y}(\boldsymbol{x}, \overline{\boldsymbol{p}}, \underline{\boldsymbol{p}})=\overline{\boldsymbol{p}}^{T}\left(\frac{\phi(\boldsymbol{x})-|\phi(\boldsymbol{x})|}{2}\right)+\underline{\boldsymbol{p}}^{T}\left(\frac{\phi(\boldsymbol{x})+|\phi(\boldsymbol{x})|}{2}\right),
$$

and the upper bound is given by

$$
\bar{y}(\boldsymbol{x}, \overline{\boldsymbol{p}}, \underline{\boldsymbol{p}})=\overline{\boldsymbol{p}}^{T}\left(\frac{\phi(\boldsymbol{x})+|\phi(\boldsymbol{x})|}{2}\right)+\underline{\boldsymbol{p}}^{T}\left(\frac{\phi(\boldsymbol{x})-|\phi(\boldsymbol{x})|}{2}\right) .
$$

An optimal IPM is yielded by minimising the expected value of

$$
\delta_{y}(\boldsymbol{x}, \overline{\boldsymbol{p}}, \underline{\boldsymbol{p}})=(\overline{\boldsymbol{p}}-\underline{\boldsymbol{p}})^{T}|\phi(\boldsymbol{x})|,
$$

by solving the linear and convex optimisation problem

$$
\{\underline{\hat{\boldsymbol{p}}}, \hat{\overline{\boldsymbol{p}}}\}=\underset{\boldsymbol{u}, \boldsymbol{v}}{\arg \min }\left\{\mathbb{E}_{\boldsymbol{x}}\left[\delta_{y}(\boldsymbol{x}, \boldsymbol{v}, \boldsymbol{u})\right]: \underline{y}\left(\boldsymbol{x}_{i}, \boldsymbol{v}, \boldsymbol{u}\right) \leq y_{i} \leq \bar{y}\left(\boldsymbol{x}_{i}, \boldsymbol{v}, \boldsymbol{u}\right), \boldsymbol{u} \leq \boldsymbol{v}\right\},
$$

where $\boldsymbol{x}_{i}$ and $y_{i}$ for $i=1, \ldots, N$ are training data points, which in the case of a metamodel should be sampled from the full model. The constraints ensure that all data points to be fitted lie within the bounds and that the upper bound is greater than the lower bound. This combination of objective function and constraints is linear and convex [7. In this work all Interval Predictor Models have polynomial bases, i.e. $\phi(\boldsymbol{x})=\left[1, \boldsymbol{x}^{i_{2}}, \boldsymbol{x}^{i_{3}}, \ldots\right]$ with $\boldsymbol{x}=\left[x_{a}, x_{b}, \ldots\right]$ and $\boldsymbol{i}_{j}=\left[i_{j, a}, i_{j, b}, \ldots\right]$ with $\boldsymbol{i}_{j} \neq \boldsymbol{i}_{k}$ for $j \neq k$.

\subsubsection{Confidence-Reliability Analysis}

The reliability $R$ of an IPM, i.e. the probability that a future unobserved data point is contained within the IPM, is bounded by

$$
\operatorname{Prob}_{\mathbb{P}^{n}}[R \geq 1-\epsilon]>1-\beta,
$$

where $\epsilon$ is the complementary confidence parameter and $\beta$ is the complementary reliability parameter which refers to the probability $\mathbb{P}^{n}$ of observing a set of $n$ 'bad' constraints (i.e. data points) such that $R<1-\epsilon$. The equation yielding $\epsilon$ 
This manuscript version is made available under the CC-BY-NC-ND 4.0

license http://creativecommons.org/licenses/by-nc-nd/4.0/

(1)2019.

and $\beta$ changes depending on whether the sequence of data points is identically and independently distributed or not.

When the data sequence is independently distributed $\epsilon$ and $\beta$ are given by

$$
\beta \geq\left(\begin{array}{c}
k+d-1 \\
k
\end{array}\right) \sum_{i=0}^{k+d-1}\left(\begin{array}{c}
N \\
i
\end{array}\right) \epsilon^{i}(1-\epsilon)^{N-i},
$$

where $N$ is the number of training data points, $k$ is the number of data points discarded by some algorithm and $d$ is the number of optimisation variables [7]. The number of data points required to give a particular $\epsilon$ and $\beta$ is given by

$$
N \leq\left\lfloor\frac{2}{\epsilon} \ln \left(\frac{1}{\beta}\right)+2 d\left(1+\frac{1}{\epsilon} \ln \left(\frac{2}{\epsilon}\right)\right)\right\rfloor,
$$

where $\lfloor$.$\rfloor is the floor operator [5]. Eqn. (11) can be inverted in order to obtain$ a more optimistic bound.

The robustness of an IPM can be evaluated by plotting $1-\epsilon$ against $1-\beta$, which will be referred to as a confidence-reliability plot, and then finding $1-\epsilon$ for an arbitrarily high value of $1-\beta$. In simple terms, if the area under the confidence-reliability plot is larger then the IPM is more robust. Quantitatively, as the confidence $(1-\beta)$ approaches 1 , the certainty on the bound on the fraction of data points contained by the IPM, $1-\epsilon$, increases.

\subsection{Probability Boxes}

Probability boxes enable the expression of epistemic uncertainty in a probability distribution 12 . Probability boxes can be seen as a generalisation of CDFs and intervals. Two types of probability boxes are possible. Firstly, a distributionfree probability box consists of an envelope made from two CDFs. Any CDF contained within the envelope is permitted. Distributional probability boxes consist of a conventional probability distribution where at least one parameter of the distribution is given as an interval rather than a crisp value. Clearly it is possible to convert distributional probability boxes to distribution free probability boxes but not vice versa.

Probability boxes are a specific case of a random set [8, 2, and therefore can be represented by a vector of epistemic uncertain parameters which fall within the unit hyper-rectangle $\boldsymbol{\theta} \in \Theta$ and an aleatory vector of parameters $\boldsymbol{\alpha}$. Their propagation through models is complicated, since there is no distribution from which to sample the epistemic parameters. Hence, conventional Monte Carlo simulation cannot be applied, as the result of propagation through the model is a probability box and not simply a CDF. Two methods are commonly used to propagate probability boxes: Double Loop Monte Carlo (sometimes referred to as search or optimisation of the epistemic space) and integration of the aleatory variables. This paper focuses on Double Loop Monte Carlo simulation, and in the following section methods are proposed to bound the reliability of a Naïve Double Loop Monte Carlo simulation, where an uninformative distribution is 
This manuscript version is made available under the CC-BY-NC-ND 4.0

license http://creativecommons.org/licenses/by-nc-nd/4.0/

○(2019.

imposed on the epistemic parameters in order to search for the maximum and minimum values (as in the brute force algorithm in [23]). The Naïve Double Loop Monte Carlo algorithm is briefly described in Algorithm 1 In this example it is assumed that $g$ has been transformed with a Copula, so that its aleatory inputs may be sampled from uniform distributions (the function $\boldsymbol{x}_{i}=T\left(\boldsymbol{\alpha}_{j}, \boldsymbol{\theta}_{i}\right)$ will be used to map between the epistemic and aleatory spaces and the true system variables). This does not justify the use of a uniform distribution over the epistemic space. Instead, this is used as a brute force optimisation tool (maxima and minima are taken over the epistemic samples, and not expectations). In reality brute force approaches to global optimisation are often impractical in high dimensional spaces, and are mainly useful as an conceptual illustration.

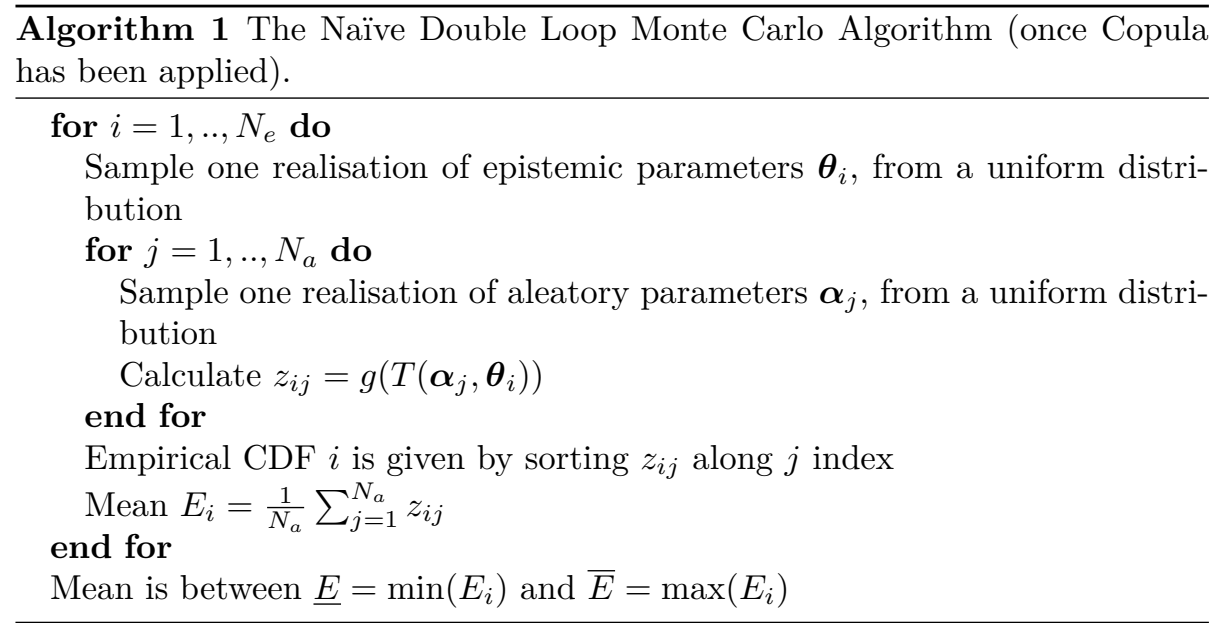

\section{Proposed Approaches: Obtaining bounds on the failure probability}

In the following sections the double loop Monte Carlo approach is used. Section 3.1 proposes a novel technique to reduce the computational costs of the naïve double loop Monte Carlo approach, where the epistemic and aleatory variables are sampled independently. Section 3.2 proposes a slight modification of the algorithm in Section 3.1 - the metamodel is trained on propagated focal elements from the traditional double loop approach. Section 3.3 proposes performing Monte Carlo simulation on the upper and lower bounds of a IPM metamodel for the performance function, inside an importance sampling optimisation loop, i.e. double loop Monte Carlo simulation. The authors stress that importance sampling for imprecise probabilities without using IPMs was already introduced in $[13$. Which approach should be used is dependant on the type of problem being studied. The naïve Monte Carlo approach is best suited to distribution free probability boxes as it avoids the need for an optimisation 
This manuscript version is made available under the CC-BY-NC-ND 4.0

license http://creativecommons.org/licenses/by-nc-nd/4.0/

○ 2019 .

loop.

Both of the discussed approaches make no assumptions about the functional form of the model, and as such the model can truly be treated as a black box including stochastic system models with an unknown noise structure. In addition, the samples required to train the metamodels may be collected in parallel since the model is not built sequentially or by optimising the performance function - the proposed approach relies exclusively on sampling the performance function.

The proposed methods are flexible and can account for epistemic uncertainty contained inside the limit state function. For example, this could be the case if there were several feasible models but a probability could not be associated with each model. This is a feature which, to the best of the authors' knowledge, no existing methods of this type can deal with. Interval failure thresholds can be used with either algorithm without an increase in the number of evaluations of the full model, since the indicator function must be monotonic with respect to changes in the threshold used to define the limit state function.

\subsection{Approach 1: Metamodels for Naïve Double Loop Ap- proach}

It is assumed that the system variables can be written as a function of separate epistemic uncertain variables falling in the unit hyper-rectangle, $\boldsymbol{\theta}$, and aleatory uncertain variables, $\boldsymbol{\alpha}$, which are uniformly distributed between 0 and 1. $\quad N$ samples are drawn of $\boldsymbol{\alpha}$ and $\boldsymbol{\theta}$, both from a uniform distribution between 0 and 1 . Samples of the performance function can then be calculated by transforming the aleatory and epistemic variables into the actual variables of the problem. Then an IPM is trained with the aleatory variables as inputs and the performance function as the output, i.e. Eqn. (9) is solved whilst replacing $\boldsymbol{x}_{i}$ with $\boldsymbol{\alpha}_{i}$ and $y\left(\boldsymbol{x}_{i}\right)$ with $g\left(T\left(\boldsymbol{\alpha}_{i}, \boldsymbol{\theta}_{i}\right)\right)$. This IPM is an uncertain model of the performance function as a function of the aleatory input variables; the epistemic uncertainties are now represented as the uncertainty in the IPM. The upper and lower bounds on the failure probability can then be calculated with minimal computational expense by performing Monte Carlo simulation on the polynomial upper and lower bounds of the performance function from the IPM, by sampling $\alpha$ uniformly between 0 and 1 and then calculating $\bar{g}(\boldsymbol{\alpha})$ and $g(\boldsymbol{\alpha})$ for the samples. A diagram of the algorithm is shown in Fig. 1 .

In contrast to the random set approach applied to the propagation of distribution free probability boxes in the multi-level metamodel algorithm [27, the approach proposed in this paper does not require multiple levels of metamodeling, since one IPM is sufficient to obtain both the upper and lower bound of the performance function. Therefore the algorithm proposed in this paper is effectively a single loop approach, as the optimisation takes place during the creation of the metamodel. If the approach is applied to distributional probability boxes then the bounds will not be tight, since random set theory based approaches overestimate the bounds when applied to problems with distributional probability boxes $[3]$. 
This manuscript version is made available under the CC-BY-NC-ND 4.0

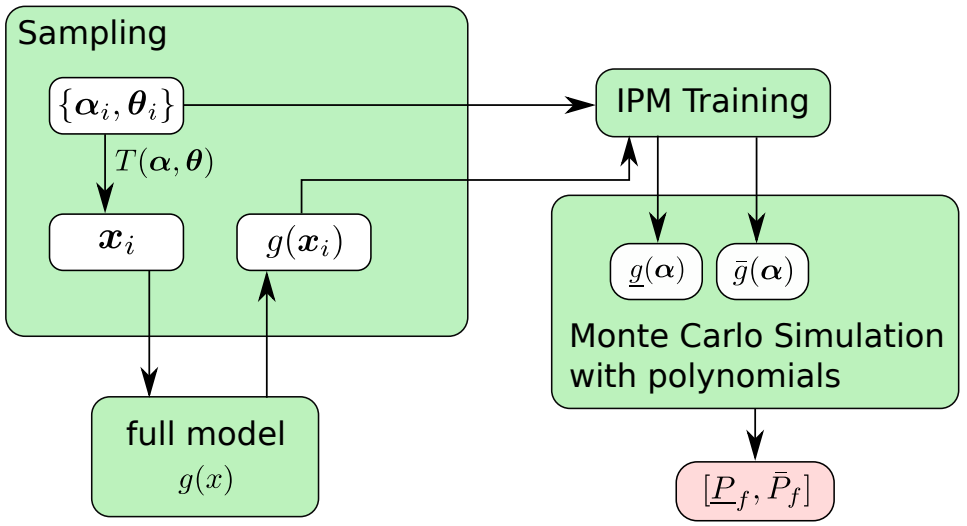

Figure 1: Approach 1: Diagram of algorithm to obtain bounds $P_{f}$ by constructing metamodels for Naïve double loop approach), by modelling the performance function in the aleatory space.

\subsection{Approach 2: IPMs trained on propagated focal ele- ments}

A focal element $\alpha_{i}$ is simply a sampled interval from a probability box. When $N_{a}$ samples of $i$ are made, for each $\boldsymbol{\alpha}_{i}$ the corresponding focal element becomes $\left[\min _{\boldsymbol{\theta} \in \Theta} T\left(\boldsymbol{\alpha}_{i}, \boldsymbol{\theta}\right), \max _{\boldsymbol{\theta} \in \Theta} T\left(\boldsymbol{\alpha}_{i}, \boldsymbol{\theta}\right)\right]$. When this is propagated through $g(\boldsymbol{x})$ the associated output will be the interval $\left.\left[\min _{\boldsymbol{\theta} \in \Theta} g\left(T\left(\boldsymbol{\alpha}_{i}, \boldsymbol{\theta}\right)\right), \max _{\boldsymbol{\theta} \in \Theta} g\left(T\left(\boldsymbol{\alpha}_{i}, \boldsymbol{\theta}\right)\right)\right]\right)$. It is trivial to train an IPM with inputs $\boldsymbol{\alpha}_{i}$, and output $\left[\min _{\boldsymbol{\theta} \in \Theta} g\left(T\left(\boldsymbol{\alpha}_{i}, \boldsymbol{\theta}\right)\right)\right.$, $\left.\left.\max _{\boldsymbol{\theta} \in \Theta} g\left(T\left(\boldsymbol{\alpha}_{i}, \boldsymbol{\theta}\right)\right)\right]\right)$. However, obtaining the focal elements for training is more expensive than propagating single values of $\boldsymbol{x}$ since the maximisation over $\boldsymbol{\theta}$ requires multiple evaluations of $g(\boldsymbol{x})$. If analytic gradients of the performance function are available then approximate focal elements can be obtained in a reduced computational time by using a Taylor series model. In this paper, focal elements for training by approximating $\left[\min _{\boldsymbol{\theta} \in \Theta} g\left(T\left(\boldsymbol{\alpha}_{i}, \boldsymbol{\theta}\right)\right), \max _{\boldsymbol{\theta} \in \Theta} g\left(T\left(\boldsymbol{\alpha}_{i}, \boldsymbol{\theta}\right)\right)\right]$ are obtained with brute force sampling, [min $\left.\min _{\boldsymbol{\theta}_{i} \in\left\{\boldsymbol{\theta}_{1}, \ldots, \boldsymbol{\theta}_{N_{e}}\right\}} g\left(T\left(\boldsymbol{\alpha}_{i}, \boldsymbol{\theta}\right)\right), \max _{\boldsymbol{\theta}_{i} \in\left\{\boldsymbol{\theta}_{1}, \ldots, \boldsymbol{\theta}_{N_{e}}\right\}} g\left(T\left(\boldsymbol{\alpha}_{i}, \boldsymbol{\theta}\right)\right)\right]$, where $\left\{\boldsymbol{\theta}_{1}, \ldots, \boldsymbol{\theta}_{N_{e}}\right\}$ are sampled by imposing a uniform distribution on the unit hypercube. Latin Hybercube Sampling or Sobol Sequence Sampling could also be used to sample $\boldsymbol{\theta}_{i}$. Then, once the IPM has been trained, the estimation of $P_{f}$ proceeds in the same way as approach 1 (independent sampling), by numerically integrating the IPM bounds (see Section 3.1). A diagram of the algorithm is shown in Fig. 2

\subsection{Approach 3: Metamodels for Non-Naïve Approach}

It is also possible to directly construct an IPM metamodel of $g(\boldsymbol{x})$, which can then be used to find bounds on $P_{f}$. The metamodel should be constructed by collecting samples of $g(\boldsymbol{x})$, by sampling $\boldsymbol{x}$ from a composite distribution which has standard deviation roughly equal to the spread of the probability 
This manuscript version is made available under the CC-BY-NC-ND 4.0

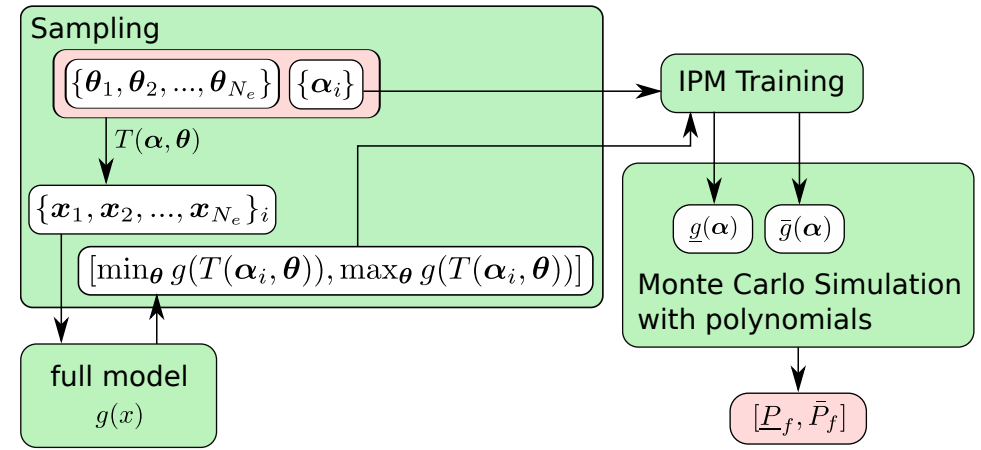

Figure 2: Approach 2: Diagram of algorithm to obtain bounds $P_{f}$ by constructing metamodels for focal element propagation, by modelling the performance function in the aleatory space.

box. Then $\bar{P}_{f}$ can be obtained from Monte Carlo simulation on $\underline{g}(\boldsymbol{x})$, and vice versa. The samples used for Monte Carlo simulation of $\bar{P}_{f}$ should be drawn from the proposal distribution and then re-weighted using importance sampling, as proposed in 28, [13]. Therefore the upper bound on the failure probability can be obtained by evaluating

$$
\bar{P}_{f}=\max _{\boldsymbol{\theta} \in \Theta} \int \bar{I}_{f}(\boldsymbol{x}) \frac{f_{X}(\boldsymbol{x}, \boldsymbol{\theta})}{h_{X}(\boldsymbol{x})} h_{X}(\boldsymbol{x}) d \boldsymbol{x},
$$

where $h_{X}(\boldsymbol{x})$ is the proposal distribution, which is used to generate training samples for the IPM from the full model, $\bar{I}_{f}(\boldsymbol{x})$ is the lower bound on the indicator function obtained from the IPM (returning 1 when $g(\boldsymbol{x}) \leq 0$, and 0 otherwise), and $f_{X}(\boldsymbol{x}, \boldsymbol{\theta})$ is a particular distribution contained by the distributional probability box. A diagram of the algorithm is shown in Figure 3

The IPM metamodel is a useful addition to vanilla importance sampling because now the optimisation in Eqn. 13 is now being performed on a continuous function, even if the performance function used is not smooth, or if a set of performance functions are being analysed. [30] shows that importance sampling results in a consistent estimator when the failure probability is continuous in the epistemic uncertain parameters.

\subsection{Confidence Bounds on Failure Probability}

In each of the proposed algorithms the interpretation of the confidence-reliability plot (described in Section 2.1.1) is different. To recap, in general, the confidencereliability plot is interpreted as the confidence that a sample of the performance function which was not present in the training set falls inside the bounds of the IPM.

When only aleatory uncertainty is present in the system inputs, i.e. random variables are used as opposed to probability boxes, the violation probability of 
This manuscript version is made available under the CC-BY-NC-ND 4.0

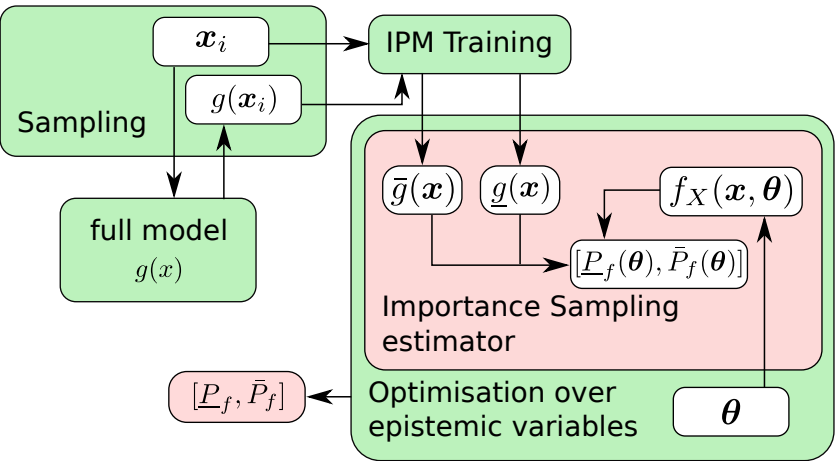

Figure 3: Approach 3: Diagram of algorithm to obtain bounds $P_{f}$ by constructing metamodels for Non-Naïve Approach, by applying importance sampling to the metamodel. Using the IPM, the importance sampling estimator produces bounds on the failure probability for a particular $\boldsymbol{\theta}$, which can be optimised over $\boldsymbol{\theta}$ to yield the true bounds on the failure probability.

the IPM is the maximum possible uncertainty in the obtained bounds on the failure probability. This is the interpretation of the confidence-reliability plot for re-weighting (Approach 3) - the confidence bound applies to the Monte Carlo estimator for the failure probability at the proposal distribution. For focal element propagation (Approach 2), if the number of aleatory samples is $N_{a}$, then obtaining the confidence reliability plot using $N_{a}$ provides the confidence in the propagation of aleatory uncertainty, because the IPM's training constraints become set inclusion relations for the focal elements (this reliability will be known as $R_{\mathrm{a}}$ ).

In the opposing case of only epistemic uncertainty, i.e. all input uncertainty can be written as intervals, the bound violation probability refers to the probability that the next sample in the brute force optimisation of the epistemic space falls outside the bounds. This interpretation can be applied to the brute force propagation of the focal elements in Approach 2 by setting $d=2$ and using the number of epistemic samples $N=N_{e}$. Alternatively, it is well known that the maximum and minimum of a sample can be used to produce a prediction interval with reliability $\frac{N_{e}-1}{N_{e}+1}$ [31, and this is the approach that will be used to determine the epistemic propagation reliability $\left(R_{\mathrm{e}}\right)$ for focal element propagation (Approach 2).

When the probability box is sampled in Approach 1, i.e. there are a mix of epistemic and aleatory variables sampled independently, the reliability of the IPM is not associated solely with epistemic or aleatory uncertainty propagation, but rather a hybrid of the two. However, it is still clear that the obtained bounds become more trustworthy when the reliability of the IPM improves.

All reliabilities $(R)$ quoted in the numerical examples for this paper are obtained by finding the reliability where the confidence is greater than 0.999 (i.e. $\beta=0.001$ ). 
This manuscript version is made available under the CC-BY-NC-ND 4.0

license http://creativecommons.org/licenses/by-nc-nd/4.0/

(O)2019.

\subsection{When is creating an IPM surrogate worthwhile?}

Readers may rightly question why creating a surrogate model is useful when efficient techniques such as re-weighting already exist [13, 29. Here simple equations are provided to motivate the use of IPMs as surrogates for calculating bounds on $P_{f}$.

Assuming that a deterministic performance function is being modelled, and $d$ is chosen such that the function is modelled perfectly such that there is no gap between the upper and lower bound of the IPM. Then the entire uncertainty in $P_{f}$ is due to the inaccuracy of the bounds as a consequence of Eqn. (11). When using a standard Monte Carlo simulation, without a metamodel, this uncertainty may be found by calculating $\bar{P}_{f}-\underline{P}_{f}$, where the bounds of the probability of failure are found by solving

$$
\sum_{i=0}^{N-N_{s}}\left(\begin{array}{c}
N \\
i
\end{array}\right)\left(1-\underline{P}_{f}\right)^{i} \underline{P}_{f}^{N-i}=\frac{\beta}{2}
$$

and

$$
\sum_{i=N-N_{s}}^{N}\left(\begin{array}{c}
N \\
i
\end{array}\right)\left(1-\bar{P}_{f}\right)^{i} \bar{P}_{f}^{N-i}=\frac{\beta}{2}
$$

which are the two-sided binomial confidence bounds on the success probability parameter for a particular confidence $\beta$ [17, where $N_{s}$ is the number of samples observed inside the failure region. If the value of $\bar{P}_{f}-\underline{P}_{f}$ is larger than the $\epsilon$ obtained from Eqn. (11) then clearly there is value in using an IPM metamodel. In the authors' experience this is usually the case when $d$ is small. It is also worth noting that for the IPM $\epsilon$ does not depend on the probability of failure. Clearly if the performance function being analysed is less complex then the uncertainty in the calculated probability of failure will be lower, as a lower $d$ can be used. This principle explains the scaling of the proposed method to high dimensional spaces, as typically functions in high dimensional spaces also require a higher $d$ as the function requires more effort to approximate correctly. In [1] an iterative method is proposed to reduce $d$ as much as possible to lessen the curse of dimensionality for the proposed method.

Having a surrogate model is useful in engineering analysis as it can be repurposed for something else besides the original analysis. IPMs at their core simply provide a method of creating surrogate models with few assumptions.

\section{Techniques to improve the efficiency of the proposed algorithms}

In the previous section it was demonstrated that the tightness and robustness of the bounds on $P_{f}$ depend on the properties of the trained IPM. Therefore in this section techniques are described to create IPMs which more tightly and robustly approximate performance functions. 
This manuscript version is made available under the CC-BY-NC-ND 4.0

license http://creativecommons.org/licenses/by-nc-nd/4.0/

○ 2019 .

\subsection{Modified Objective Function}

Since the purpose of structural reliability analysis is to obtain the failure probability, which is calculated by integration of an indicator function, the priority should be to model the performance function as accurately as possible where it is close to zero [1. This is not achieved in regular Interval Predictor Models because the objective function $\left(\mathbb{E}_{\boldsymbol{x}}\left[\delta_{y}(\boldsymbol{x}, \boldsymbol{v}, \boldsymbol{u})\right]\right)$ minimises the expectation of the width of the IPM everywhere in the data containing region. A hyperbolic tangent objective function $(w(\boldsymbol{x}, \boldsymbol{v}, \boldsymbol{u}))$ can be used to remedy the problem, which is given by

$$
w(\boldsymbol{x}, \boldsymbol{v}, \boldsymbol{u})=\sum_{i} \tanh \left(b \bar{y}\left(\boldsymbol{x}_{i}, \boldsymbol{v}, \boldsymbol{u}\right)\right)-\tanh \left(b \underline{y}\left(\boldsymbol{x}_{i}, \boldsymbol{v}, \boldsymbol{u}\right)\right),
$$

where $b$ is a positive real scaling factor which can be increased or decreased to alter the convergence of the IPM. Increasing $b$ will reduce the objective function to the indicator function - i.e. the IPM will behave more like a classifier. In fact, since the proposed objective function is an expectation, it will be equal to twice the difference between the empirically computed bounds on the probability of failure $\left(\underline{P}_{f}-\bar{P}_{f}\right)$. This loss function is particularly useful whenever a metamodel is created for the purposes of reliability analysis. Decreasing $b$ will result in a linear scaling between the objective and $y(\boldsymbol{x})$, i.e. similar to the unmodified objective function. This function is advantageous because when the IPM is close to zero there is a clear incentive to make the model as tight as possible here. Furthermore the function is smooth and analytically differentiable with respect to the parameter vector, which permits easier optimisation, and therefore its gradient can be obtained as

$$
\begin{aligned}
\nabla_{\boldsymbol{u}} w(\boldsymbol{x}, \boldsymbol{v}, \boldsymbol{u})=\sum_{i} b \frac{\phi\left(\boldsymbol{x}_{i}\right)-\left|\phi\left(\boldsymbol{x}_{i}\right)\right|}{2} \operatorname{sech}^{2}\left(b \bar{y}\left(\boldsymbol{x}_{i}, \boldsymbol{v}, \boldsymbol{u}\right)\right)- \\
\sum_{i} b \cdot \frac{\phi\left(\boldsymbol{x}_{i}\right)+\left|\phi\left(\boldsymbol{x}_{i}\right)\right|}{2} \operatorname{sech}^{2}\left(b \cdot \underline{y}\left(\boldsymbol{x}_{i}, \boldsymbol{v}, \boldsymbol{u}\right)\right),
\end{aligned}
$$

and

$$
\begin{aligned}
\nabla_{\boldsymbol{v}} w(\boldsymbol{x}, \boldsymbol{v}, \boldsymbol{u})=\sum_{i} b \cdot \frac{\phi\left(\boldsymbol{x}_{i}\right)+\left|\phi\left(\boldsymbol{x}_{i}\right)\right|}{2} \operatorname{sech}^{2}\left(b \cdot \bar{y}\left(\boldsymbol{x}_{i}, \boldsymbol{v}, \boldsymbol{u}\right)\right)- \\
\sum_{i} b \cdot \frac{\phi\left(\boldsymbol{x}_{i}\right)-\left|\phi\left(\boldsymbol{x}_{i}\right)\right|}{2} \operatorname{sech}^{2}\left(b \cdot \underline{y}\left(\boldsymbol{x}_{i}, \boldsymbol{v}, \boldsymbol{u}\right)\right) .
\end{aligned}
$$

tanh is a non-convex function, and therefore one may wish to define a convex approximation of the function for practical purposes. In this paper the approximation

$$
\left.\tanh \left(\bar{y}\left(\boldsymbol{x}_{i}, \boldsymbol{v}, \boldsymbol{u}\right)\right)-\tanh \left(\underline{y}\left(\boldsymbol{x}_{i}, \boldsymbol{v}, \boldsymbol{u}\right)\right) \approx w_{i} *\left(\bar{y}\left(\boldsymbol{x}_{i}, \boldsymbol{v}, \boldsymbol{u}\right)\right)-\underline{y}\left(\boldsymbol{x}_{i}, \boldsymbol{v}, \boldsymbol{u}\right)\right),
$$

is used, with $w_{i}=\frac{\tanh y_{i}}{y_{i}}$. In other words, the original IPM loss is re-weighted when the data is close to the limit state surface, whilst the loss remains a linear 
This manuscript version is made available under the CC-BY-NC-ND 4.0

license http://creativecommons.org/licenses/by-nc-nd/4.0/

(1)2019.

function of the IPM parameters. This approximation will only be reasonable when the data has low noise in $y$.

\subsection{Wait and judge approach}

The bound in Eqn. (11) may be overly conservative as it relies upon the assumption that the number of support constraints (i.e. the number of constraints which when removed result in a tighter IPM) is less than the number of optimisation variables. In fact, once the IPM is created the number of support constraints can be measured and then the Wait and Judge approach to scenario optimisation can be applied to obtain a better bound on the reliability of the IPM [4]. The improved bound is given by letting $\epsilon$ be a function of the number of support constraints $s_{N}^{*}$ such that $\epsilon\left(s_{N}^{*}\right)=1-t\left(s_{N}^{*}\right)$. Then for $0<\beta<1$ and $0<s_{N}^{*}<d$ the equation

$$
\frac{\beta}{N+1} \sum_{m=k}^{N}\left(\begin{array}{c}
m \\
k
\end{array}\right) t^{m-k}-\left(\begin{array}{c}
N \\
k
\end{array}\right) t^{N-k}=0
$$

has one solution, $t\left(s_{N}^{*}\right)$ in the interval $[0,1]$. When the optimisation program being studied is non-convex $\epsilon$ is obtained from

$$
\epsilon\left(s_{N}^{*}\right)= \begin{cases}1, & \text { for } s_{N}^{*}=N \\ 1-N-s_{N}^{*} \sqrt{\frac{\beta}{N\left(s_{N}^{*}\right)},}, & \text { otherwise. }\end{cases}
$$

\section{Numerical Examples}

\subsection{Cantilever Beam}

\subsubsection{Problem Description}

For the simple example of a cantilever beam with a point load, $F$, at any point on the beam, the maximum deflection of the end of the beam is given by

$$
\delta_{\max }=\frac{F a^{2}}{6 E I}(3 l-a)
$$

where $I$ is the moment of inertia of the beam, $a$ is the distance of the point load from the fixed end of the beam, $l$ is the length of the beam and $E$ is the modulus of elasticity of the beam [14. $E, I$ and $a$ were fixed and $l$ and $F$ were given by distributional probability boxes with normal distributions and uncertain means (Case A). The chosen values of the parameters are shown in Table 1 . In order to demonstrate the application of Approach 1 (independent sampling) to a problem where epistemic uncertainty is more influential, the analysis was repeated using epistemic uncertainty also in the standard deviation of the random variables (Case B). It was assumed that the beam 'fails' when the maximum deflection is greater than $35 \mathrm{~mm}$. 
This manuscript version is made available under the CC-BY-NC-ND 4.0

license http://creativecommons.org/licenses/by-nc-nd/4.0/

(1)2019.

The independent sampling method described in Section 3.1 for the naïve double loop approach was used to find the probability of failure of the system, by creating a polynomial IPM of maximum degree $1\left(\phi\left(\alpha_{l}, \alpha_{F}\right)=\left[1, \alpha_{l}, \alpha_{F}, \alpha_{l} \alpha_{F}\right]\right)$ of the performance function. In total 1000 samples of the true model were made. The focal element propagation method in Section 3.2 was applied with the same basis.

This was compared to the re-weighting approach described in Section 3.3 A normal proposal distribution was used with $\mu_{F}=30500, \sigma_{F}=230, \mu_{l}=5050$, $\sigma_{l}=230$. Again, a polynomial IPM of maximum degree $1(\phi(l, F)=[1, l, F, l F])$ was trained with 1000 samples. The bounds on the probability of failure were obtained by using MATLAB's fmincon function on the failure probability reweighted estimator.

\begin{tabular}{||ccccc||}
\hline Variable & Distribution & Mean & Std (Case A) & Std (Case B) \\
\hline \hline$E$ & Fixed & $200000 \mathrm{~N} / \mathrm{mm}^{2}$ & N/A & N/A \\
$I$ & Fixed & $78125000 \mathrm{~mm}^{4}$ & N/A & N/A \\
$l$ & Normal & {$[5000,5100] \mathrm{mm}$} & $200 \mathrm{~mm}$ & {$[200,220] \mathrm{mm}$} \\
$a$ & Fixed & $3000 \mathrm{~mm}$ & $\mathrm{~N} / \mathrm{A}$ & $\mathrm{N} / \mathrm{A}$ \\
$F$ & Normal & {$[30000,31000] \mathrm{N}$} & $200 \mathrm{~N}$ & {$[200,220] \mathrm{N}$} \\
\hline
\end{tabular}

Table 1: Values of input variables for cantilever beam problem.

The confidence-reliability analysis was then performed using Eqn. (11).

\subsubsection{Results}

The metamodel for the performance function with a polynomial IPM of maximum degree 1 is shown in Fig. 4 The reference solution $\left(P_{f}=[0.40,0.81]\right)$ was obtained by naïve Monte Carlo simulation with a large number of samples. Fig.6 shows the confidence-reliability analysis (calculated with Eqn. (11)) for the calculation of $P_{f}$ using the IPM in Fig. 4 , corresponding to a reliability of approximately 0.98 with high confidence (0.999). This IPM has 6 support constraints and hence the bound on $R$ is fairly tight. Inverting Eqn. (11), it was calculated that 19619 samples would be required to obtain $\beta=0.001$ and $\epsilon=0.001$. Fig. 5 shows the bounds of the CDF which were computed by Monte Carlo analysis of the performance functions shown in Fig. 4 . The bounds on $P_{f}$ can be tightened by increasing the number of training samples which allows the degree of the IPM to be increased without decreasing the bound on $R$. For example, by taking 2000 samples and using the loss function in Eqn. $(19), P_{f}=[0.388,0.795]$ for a polynomial IPM with basis $\phi\left(\alpha_{l}, \alpha_{F}\right)=\left[1, \alpha_{l}, \alpha_{F}, \alpha_{l} \alpha_{F}, \alpha_{l}^{2}, \alpha_{F}^{2}\right]$, where $R>0.987$ with high confidence (0.999). When using the loss function in Eqn. 16 no notable improvement was found in the bounds on $P_{f}$, since the performance function and IPM were relatively simple, and the epistemic uncertainty in $P_{f}$ is large. Fig. 9 shows the bounds of the CDF which were computed by Monte Carlo analysis of the obtained IPM, when using the input Case B. The reference solution computed with Double Loop Monte Carlo simulation was $P_{f}=[0.40,0.81]$. 
This manuscript version is made available under the CC-BY-NC-ND 4.0

license http://creativecommons.org/licenses/by-nc-nd/4.0/

○(2019.

The re-weighting strategy with the direct IPM metamodel (shown in Fig. 7) directly obtains the reference solution. Since the direct IPM is a model of a linear function it can be easily represented exactly by the IPM, and hence there are no support constraints. This allows us to bound the reliability of the IPM to at least 0.99 with high confidence $(\beta=0.001)$ by using the wait and judge approach. For similar reasons, the correct solution can be obtained with as few as 10 samples using the re-weighting estimator. Fig. 8 shows the confidencereliability analysis for the calculation of $P_{f}$ using Fig. 7

A summary of the results for this example are given in Table 2

\begin{tabular}{||llll||}
\hline Approach & $N_{\text {Samples }}$ & Case A & Case B \\
\hline \hline Reference Solution, Double Loop & $N_{a}=10^{3}, N_{e}=$ & $P_{f}=[0.4,0.81]$ & $P_{f}=[0.4,0.81]$ \\
& $10^{6} N_{e} \times N_{a}=$ & \\
Monte Carlo & $10^{9}$ & \\
& & $P_{f}=[0.36,0.81], R \geq$ & $P_{f}=[0.36,0.81], R \geq$ \\
Approach 1 (independent sam- & 1000 & 0.98 & 0.98 \\
pling, IPM degree 1) & & $P_{f}=[0.39,0.80], R \geq$ & $P_{f}=[0.39,0.80], R \geq$ \\
Approach 1 (independent sam- & 2000 & 0.99 & 0.99 \\
pling, IPM degree 2) & & $P_{f}=[0.39,0.81], R \geq$ & $P_{f}=[0.38,0.79], R \geq$ \\
Approach 1 (independent sam- & 300 & 0.94 & 0.94 \\
pling, IPM degree 1) & & $P_{f}=[0.375,0.795]$ & $P_{f}=[0.373,0.785]$ \\
Approach 2 (focal element prop- & $1000\left(N_{a}=125\right.$, \\
agation, IPM degree 1) & $\left.N_{e}=8\right)$ & $R_{\mathrm{a}}>0.85, R_{\mathrm{e}} \approx 0.78$ & $R_{\mathrm{a}}>0.85, R_{\mathrm{e}} \approx 0.78$ \\
Approach 3 (re-weighting, IPM & 1000 & $P_{f}=[0.4,0.81], R \geq$ & $P_{f}=[0.4,0.81], R \geq$ \\
degree 1) & 0.99 & 0.99 \\
Approach 3 (re-weighting, IPM & 10 & $P_{f}=[0.4,0.81], R \geq$ & $P_{f}=[0.4,0.81], R \geq$ \\
degree 1) & & 0.021 & 0.021 \\
\hline
\end{tabular}

Table 2: Summary of results for cantilever beam reliability analysis in Section 5.1 .2

\subsection{Dynamic response of a non-linear oscillator}

\subsubsection{Problem Description}

In order to demonstrate the application of the method on a non-linear performance function, the well known non-linear oscillator example is used [10] [15]. The performance function is defined by

$$
g_{\text {oscillator }}\left(C_{1}, C_{2}, M, R, T_{1}, F_{1}\right)=3 R-\left|\frac{2 F_{1}}{M \omega_{0}^{2}} \sin \left(\frac{\omega_{0} T_{1}}{2}\right)\right|,
$$

where the natural frequency of the oscillator, $\omega_{0}=\sqrt{\frac{C_{1}+C_{2}}{M}}, M$ is the mass, $C_{1}$ and $C_{2}$ are the spring constants of the primary and secondary springs, $R$ is the displacement at which the secondary spring yields, $t_{1}$ is the duration of the loading, and $F_{1}$ is the amplitude of the applied force. As usual, the 
This manuscript version is made available under the CC-BY-NC-ND 4.0

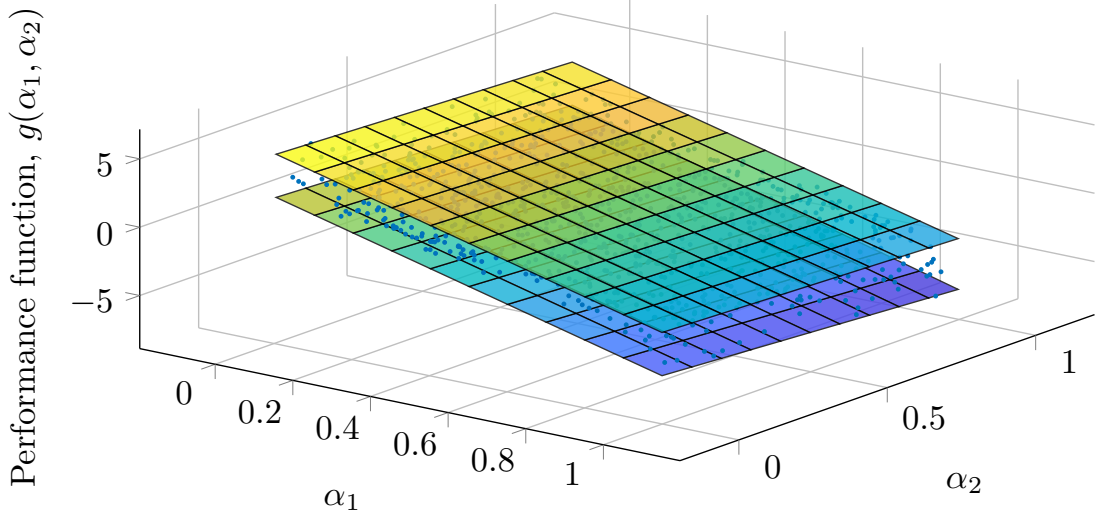

Figure 4: Degree 1 IPM of the performance function in the aleatory space for the cantilever beam, created by independently sampling the aleatory and epistemic variables (Approach 1).

system fails when $g_{\text {oscillator }} \leq 0$, hence the failure probability to be estimated is $P_{f}=\mathbb{P}\left(g_{\text {oscillator }} \leq 0\right)$. The distributions and probability boxes assigned to the inputs are listed in Table 3 A diagram of the system is shown in Fig. 10

The methods described in Section 3.1 and Section 3.2 for the naïve double loop approach were used to find the probability of failure of the system, by creating a polynomial IPM of maximum degree 1 of the performance function. In total, 1000 samples of the true model were made.

This was compared to the approach described in Section 3.3. with a normal proposal distribution with mean at the centre of the probability box and standard deviation set to cover the support of the probability box $\left(\mu_{\text {proposal }}=\frac{\bar{\mu}+\underline{\underline{\mu}}}{2}\right.$ and $\sigma_{\text {proposal }}=\sqrt{\left.\left(\frac{\bar{\mu}-\mu}{2 \times 3}\right)^{2}+\sigma^{2}\right)}$. Again, a polynomial IPM of maximum degree 1 was trained with 1000 samples. The bounds on the probability of failure were obtained by using MATLAB's fmincon function on the failure probability reweighted estimator. The performance function loss (Eqn. 16) was not used in this example, as it was not found to significantly improve the performance of the model.

\begin{tabular}{||cccc||}
\hline Variable & Distribution & Mean & Standard Deviation \\
\hline \hline$C_{1}$ & Normal & 1 & 0.1 \\
$C_{2}$ & Normal & 0.1 & 0.01 \\
$R$ & Normal & {$[0.45,0.5]$} & 0.05 \\
$M$ & Normal & 1 & 0.05 \\
$t_{1}$ & Normal & {$[0.95,1]$} & 0.2 \\
$F_{1}$ & Normal & {$[0.95,1]$} & 0.2 \\
\hline
\end{tabular}

Table 3: Values of input variables for non-linear oscillator. 
This manuscript version is made available under the CC-BY-NC-ND 4.0

Failure Threshold $=35 \mathrm{~mm}$

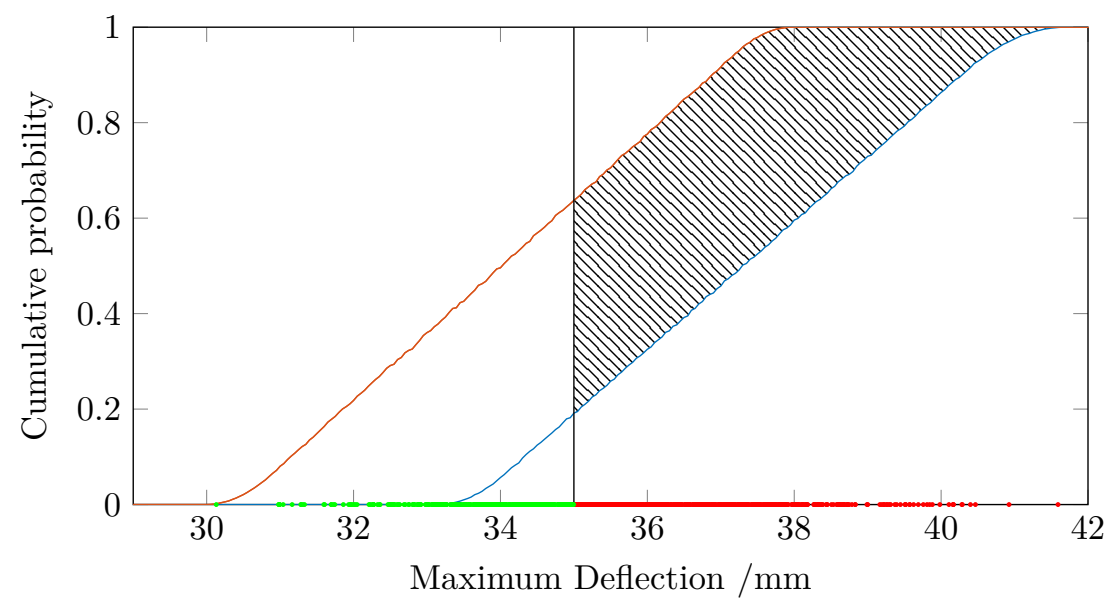

Figure 5: CDF bounds obtained by Monte Carlo analysis on the performance function modelled in Fig. 4 The 'flat' bounds are a remnant of the low degree IPM chosen to represent the performance function of the cantilever beam. Training samples are shown on the abscissa axis. 
This manuscript version is made available under the CC-BY-NC-ND 4.0

license http://creativecommons.org/licenses/by-nc-nd/4.0/

(O)2019.

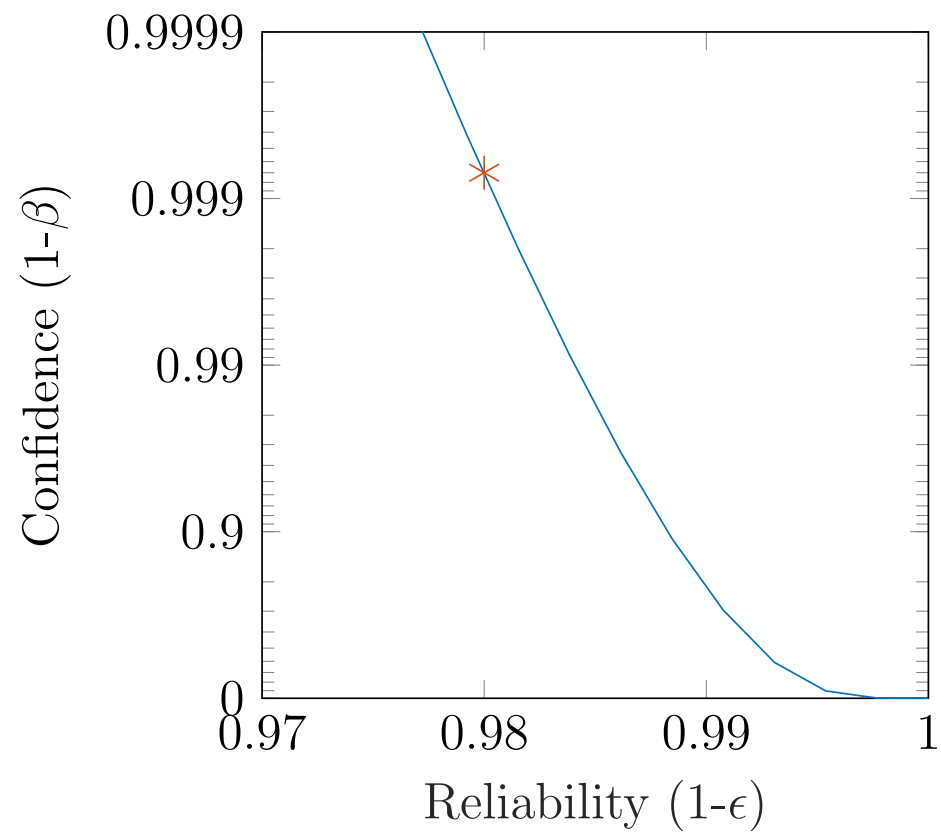

Figure 6: Confidence-reliability plot corresponding to the IPM used to model the performance function in Fig. 4 for the cantilever beam and calculate $P_{f}$ (as described in Section 2.1.1. This plot corresponds to a reliability of approximately 0.98 with confidence 0.999 , which is shown on the plot as a star. 
This manuscript version is made available under the CC-BY-NC-ND 4.0

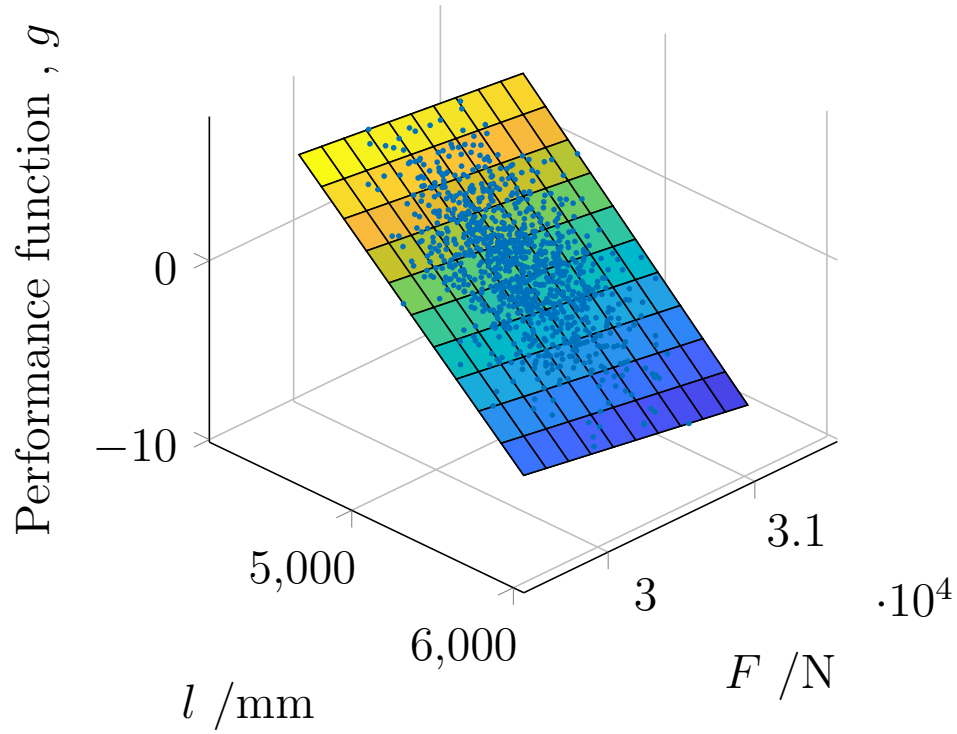

Figure 7: Direct Degree 1 IPM of the performance function for the cantilever beam, created for the re-weighting approach (Approach 3).

\subsubsection{Results}

The results for the analysis of the oscillator are shown in Table 4 including number of support constraints is shown for each trained IPM, and a bound on the reliability computed as described in Section 4.2 The reference solution was computed with naïve double loop Monte Carlo simulation, using 10000 inner loop and 10000 outer loop samples, resulting in a total of $10^{10}$ model queries.

\begin{tabular}{|c|c|c|c|c|}
\hline Approach & $N_{\text {Samples }}$ & {$\left[\bar{P}_{f}, \underline{P}_{f}\right]$} & Confidence & $s_{N}^{*}$ \\
\hline $\begin{array}{l}\text { Reference solution (double loop } \\
\text { Monte Carlo) }\end{array}$ & $10^{10}$ & 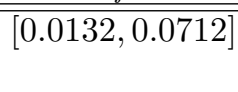 & - & - \\
\hline $\begin{array}{l}\text { Approach } 1 \text { (independent sam- } \\
\text { pling, IPM degree } 3 \text { ) }\end{array}$ & 1000 & {$[0.0138,0.0741]$} & $\begin{array}{l}R \geq 0.80, R^{*}> \\
0.87\end{array}$ & 90 \\
\hline $\begin{array}{l}\text { Approach } 1 \text { (independent sam- } \\
\text { pling, IPM degree } 2 \text { ) }\end{array}$ & 1000 & {$[0.0073,0.123]$} & $\begin{array}{l}R \geq 0.92, R^{*}> \\
0.94\end{array}$ & 33 \\
\hline $\begin{array}{l}\text { Approach } 2 \text { (focal elements, } \\
\text { IPM degree 2) }\end{array}$ & 1000 & {$[0.012,0.11]$} & $\begin{array}{l}R_{\mathrm{a}}>0.62, R_{\mathrm{e}} \approx \\
0.67, R_{\mathrm{a}}^{*}>0.76\end{array}$ & 34 \\
\hline $\begin{array}{l}\text { Approach } 3 \text { (re-weighting, IPM } \\
\text { degree 2) }\end{array}$ & 1000 & {$[0.0735,0.0114]$} & $\begin{array}{l}R \geq 0.92, R^{*}> \\
0.94\end{array}$ & 33 \\
\hline
\end{tabular}

Table 4: Summary of results for non-linear oscillator $\left(S_{N}^{*}\right.$ : maximum superior constraints, $R$ : Confidence a priori; $R^{*}$ : confidence wait and judge). 
This manuscript version is made available under the CC-BY-NC-ND 4.0

license http://creativecommons.org/licenses/by-nc-nd/4.0/

(O)2019.

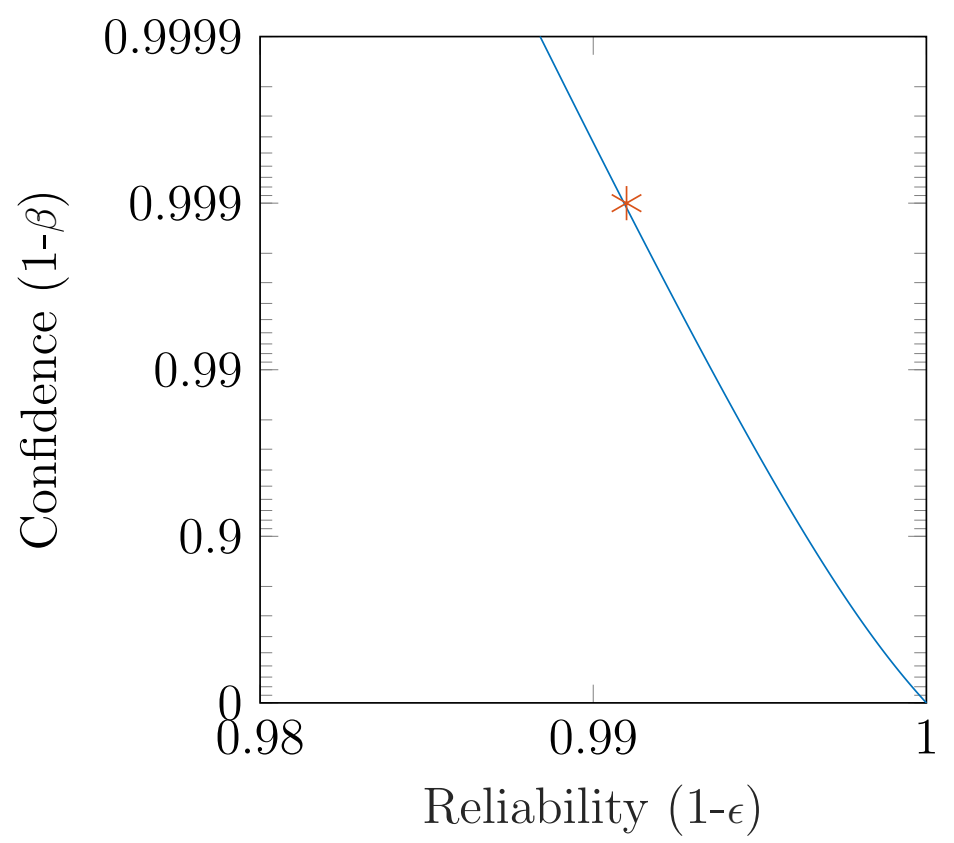

Figure 8: Confidence-reliability plot corresponding to the IPM used to model the performance function in Fig. 7 for the cantilever beam and calculate $P_{f}$. This plot corresponds to a reliability of over 0.99 with confidence 0.999 , which is shown on the plot as a star.

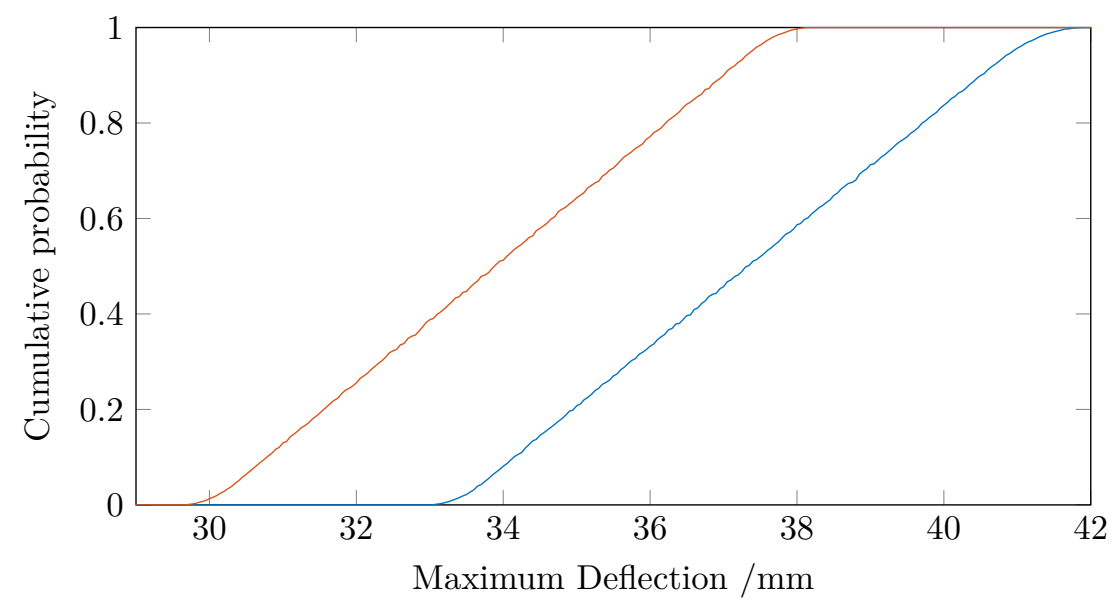

Figure 9: CDF bounds obtained by Monte Carlo analysis on the performance function modelled the IPM for the second cantilever beam input set. The 'flat' bounds are a remnant of the low degree IPM chosen to represent the performance function of the cantilever beam. 
This manuscript version is made available under the CC-BY-NC-ND 4.0
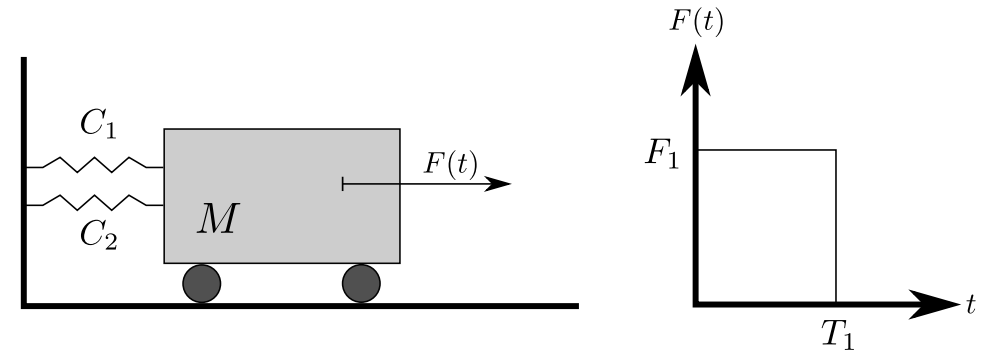

Figure 10: A non-linear oscillator. 
This manuscript version is made available under the CC-BY-NC-ND 4.0 license http://creativecommons.org/licenses/by-nc-nd/4.0/ ○ 2019 .

\begin{tabular}{||cccc||}
\hline Variable & Distribution & Mean & Standard Deviation \\
\hline \hline Young's Modulus $\times 4$ & Normal & {$[65,75] \mathrm{GPa}$} & $1.05 \mathrm{GPa}$ \\
Density $\times 4$ & Normal & {$[2500,2900] \mathrm{kg} / \mathrm{m}^{3}$} & $270 \mathrm{~kg} / \mathrm{m}^{3}$ \\
\hline
\end{tabular}

Table 5: Summary of the 8 random inputs for Satellite Model. Both of the random variables shown above are repeated for the 4 structural components of the model.

\subsection{Small satellite}

\subsubsection{Problem Description}

In this section, the developed techniques are applied to a NASTRAN model of a satellite with 900 QUAD4 elements ( 5300 DOF) [18. The model is available in the OpenCossan software [20. All DOF of the nodes at the bottom of the nozzle have been constrained by modelling the boundary conditions that the nozzle is attached to the supporting structure with bolts, and a vertical acceleration of $6 g$ has been applied together with a horizontal acceleration of $1 g$ $\left(g=9.81 \mathrm{~m} / \mathrm{s}^{2}\right)$. The structure consists of 4 components, namely nozzle, upper and lower panels, central cylinder and the vertical panels. The combined effect of the uncertainty in the young's modulus and density of each of these components on the second natural frequency is investigated, and epistemic uncertainties in these two quantities as shown in Table 5 are considered.

The method described in Section 3.1 for the naïve double loop approach was used to find bounds on the CDF for the second eigenvalue and also bounds on the expectation of the second eigenvalue, by taking 1000 samples from the full model. In order to achieve this the method was modified to build an IPM for the response of the model rather than the performance function, which is required when calculating expectations rather than probabilities of failure. Approach 2 (Section 3.2 was used to train an IPM on focal elements propagated with brute force optimisation, making 4 samples in the brute force optimisation and 250 aleatory samples (samples of $\boldsymbol{\alpha}$ ). An IPM with a polynomial basis of maximum degree 1 was used.

To obtain a reference solution, the double loop Monte Carlo method was used with 50 inner loop Monte Carlo samples, and 100 outer loop Bayesian Optimisation evaluations made by MATLAB's bayesopt routine, for both the upper and lower bound (i.e. $2 \times 100 \times 50=10000$ samples in total).

\subsubsection{Results}

For Approach 1 (independent sampling) and Approach 2 (focal element propagation), bounds on the CDF of the output are shown in Fig. 12 . Fig. 13 shows the confidence-reliability analysis for the IPM, corresponding to a reliability of approximately 0.97 with high confidence. Increasing the maximum degree of the IPM to 2 tightens the prediction interval, however the reliability of the bounds is decreased. Similar results were obtained by using a radial basis, trained 
This manuscript version is made available under the CC-BY-NC-ND 4.0

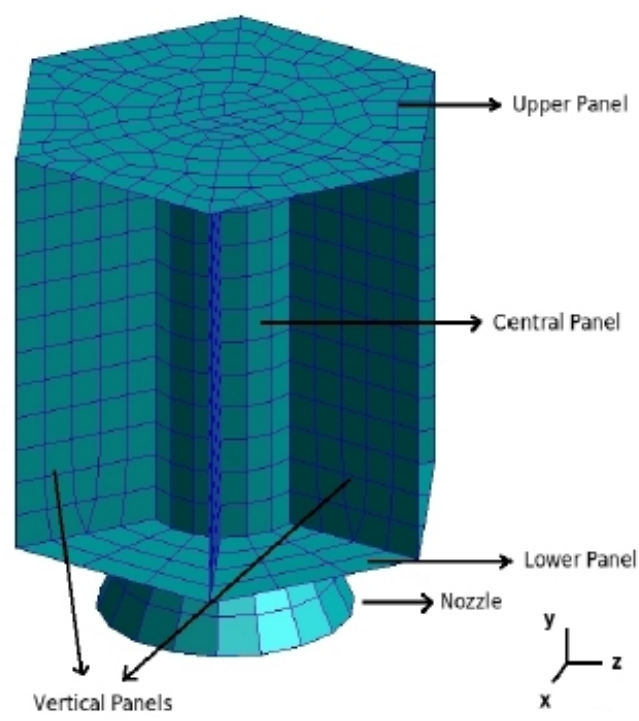

Figure 11: Small Satellite Model in NASTRAN. Full details of model available from [19].

using with the same number of terms. Applying Approach 3 (re-weighting), with a normal proposal distribution with mean at the centre of the probability box and standard deviation set to cover the support of the probability box $\left(\mu_{\text {proposal }}=\frac{\bar{\mu}+\underline{\underline{\mu}}}{2}\right.$ and $\left.\sigma_{\text {proposal }}=\sqrt{\left(\frac{\bar{\mu}-\underline{\mu}}{2 \times 3}\right)^{2}+\sigma^{2}}\right)$, similar bounds were obtained on the expectation of the 2nd eigenvalue. A summary of the results for this example are given in Table 6. Most of the results appear overly conservative, indicating the IPM is a poor fit for the model response. However, Approach 2 (IPM for focal element propagation) has impressive agreement with the reference solution. As expected, the IPM with Maximum Degree 2 has a lower reliability, and hence underestimates the upper bound of the expectation.

Computing the reference solution resulted in bounds on the expectation of the 2nd eigenvalue of $\mathbb{E}_{\boldsymbol{x}}=[359,481]$. For comparison, a double loop method with Latin Hyper Cube sampling for both loops (with 40 inner loop samples and 25 outer loop samples, resulting in 1000 total samples), was found to underestimate the interval width, $\mathbb{E}_{\boldsymbol{x}}=[367,447]$. Therefore, the authors do not recommend the use of Latin Hypercube Sampling, since in probability bounds analysis it is desirable to find the outer approximation of the interval containing the results, in order to be conservative in an engineering sense. 
This manuscript version is made available under the CC-BY-NC-ND 4.0

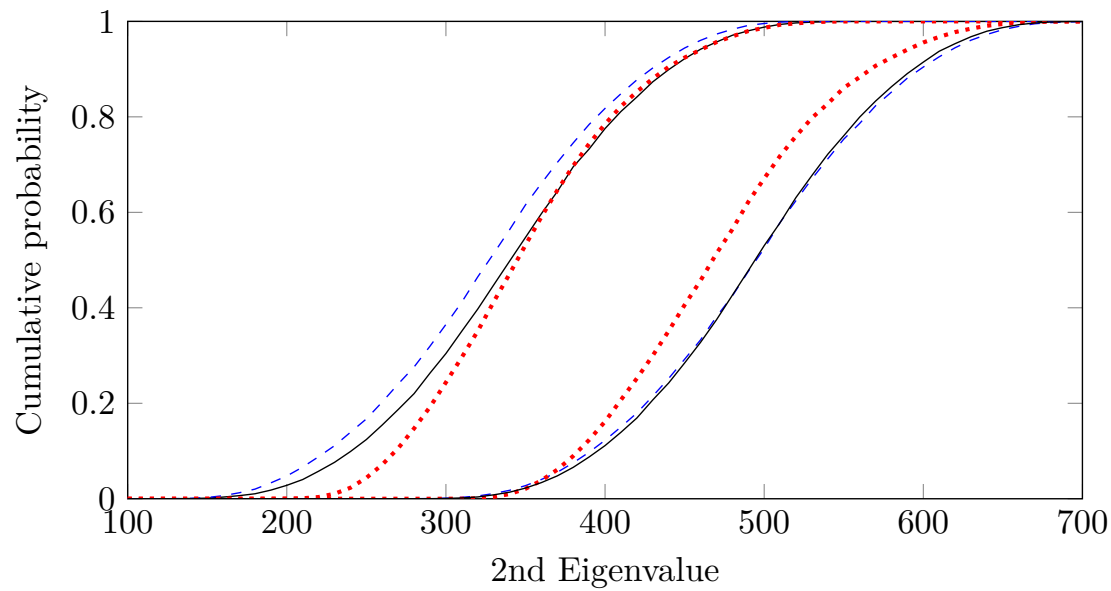

— Focal Element Propagation (Approach 2), IPM degree 1 - - Independent Sampling (Approach 1), IPM degree 1 …... Independent Sampling (Approach 1), IPM degree 2

Figure 12: CDF bounds obtained by Monte Carlo analysis on an IPM for the 2nd Eigenvalue of a small satellite (modal analysis).

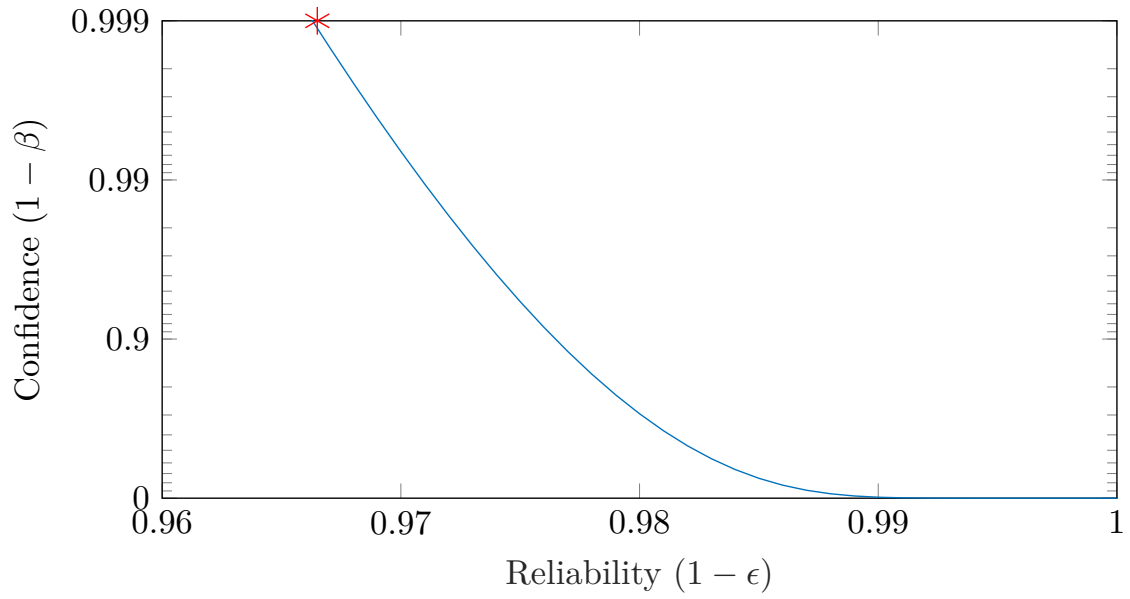

Figure 13: Confidence-reliability plot corresponding to the IPM of the 2nd Eigenvalue of small satellite modal analysis. This plot corresponds to a reliability of over 0.97 with confidence 0.999 , which is shown on the plot as a star. 
This manuscript version is made available under the CC-BY-NC-ND 4.0 license http://creativecommons.org/licenses/by-nc-nd/4.0/ (1)2019.

\begin{tabular}{|c|c|c|c|}
\hline & $N_{\text {samples }}$ & $\underline{\mathbb{E}}_{\boldsymbol{x}}, \overline{\mathbb{E}}_{\boldsymbol{x}}$ & Confidence \\
\hline Reference & 10000 & {$[359,481]$} & N/A \\
\hline Latin Hypercube Double Loop & 1000 & {$[367,447]$} & $\mathrm{N} / \mathrm{A}$ \\
\hline $\begin{array}{l}\text { Approach } 1 \text { (independent sam- } \\
\text { pling, IPM degree 1) }\end{array}$ & 1000 & {$[327,495]$} & $R \geq 0.97$ \\
\hline $\begin{array}{l}\text { Approach } 1 \text { (independent sam- } \\
\text { pling, IPM degree 2) }\end{array}$ & 2000 & {$[349,471]$} & $R \geq 0.88$ \\
\hline $\begin{array}{l}\text { Approach } 2 \text { (focal element, IPM } \\
\text { degree } 2 \text { ) }\end{array}$ & $\begin{array}{l}1000\left(N_{a}=250\right. \\
\left.N_{e}=4\right)\end{array}$ & {$[352,471]$} & $\begin{array}{l}R_{\mathrm{a}}>0.55, R_{\mathrm{e}} \approx \\
0.6\end{array}$ \\
\hline $\begin{array}{l}\text { Approach } 2 \text { (focal element, IPM } \\
\text { degree 1) }\end{array}$ & $\begin{array}{l}1000\left(N_{a}=200\right. \\
\left.N_{e}=5\right)\end{array}$ & {$[346,489]$} & $\begin{array}{l}R_{\mathrm{a}}>0.84, R_{\mathrm{e}} \approx \\
0.67\end{array}$ \\
\hline $\begin{array}{l}\text { Approach } 2 \text { (focal element, IPM } \\
\text { degree 1) }\end{array}$ & $\begin{array}{l}1000 \quad\left(N_{a}=40\right. \\
\left.N_{e}=25\right)\end{array}$ & {$[353,485]$} & $\begin{array}{l}R_{\mathrm{a}}>0.33, R_{\mathrm{e}} \approx \\
0.92\end{array}$ \\
\hline $\begin{array}{l}\text { Approach } 2 \text { (focal element, IPM } \\
\text { degree 1) }\end{array}$ & $\begin{array}{l}1000\left(N_{a}=125\right. \\
\left.N_{e}=8\right)\end{array}$ & {$[339,487]$} & $\begin{array}{l}R_{\mathrm{a}}>0.746, R_{\mathrm{e}} \approx \\
0.78\end{array}$ \\
\hline $\begin{array}{l}\text { Approach } 3 \text { (re-weighting, IPM } \\
\text { degree 1) }\end{array}$ & 1000 & {$[321,477]$} & $R \geq 0.966$ \\
\hline
\end{tabular}

Table 6: Summary of results for small satellite model using different approaches.

\section{Conclusion}

This paper proposed a computational method of bounding the reliability of the propagation of epistemic uncertainty. Novel loss functions are introduced to ensure tightness when IPMs are created from data representing performance functions. The approach proposed in this paper is applicable to the double loop Monte Carlo algorithm as well as the naïve approach where an uninformative distribution is sampled rather than using optimisation to propagate intervals. A key benefit is that the performance function is smoothed which enables easier optimisation of the probability of failure. Both of the proposed approaches do not make restrictive assumptions about the functional form of the model response, and easily parallelisable.

The authors expect future work to apply new IPM technologies to the proposed methodology to increase the data efficiency of the IPMs created, and hence obtain tighter bounds on small failure probabilities, e.g. active learning techniques.

\section{Acknowledgements}

This research has been generously supported by the EPSRC Centre for Doctoral Training in Nuclear Fission - Next Generation Nuclear (Grant reference: $\mathrm{EP} / \mathrm{L} 015390 / 1)$ which is gratefully acknowledged by the authors. This research is also partly funded by the UK Engineering \& Physical Sciences Research Council with grant no. EP/R006768/1 
This manuscript version is made available under the CC-BY-NC-ND 4.0

license http://creativecommons.org/licenses/by-nc-nd/4.0/

○ 2019 .

\section{References}

[1] D. Altieri and E. Patelli. An efficient method for estimating conditional failure probabilities. In The 8th International Workshop on Reliable Engineering Computing (REC2018), Liverpool, UK, pages 163-168, 2018. URL https://rec2018.uk/papers/REC2018-18_D.Altieri, $\% 20$ E. $\% 20$ Patelli__An $\% 20$ efficient $\% 20$ method $\% 20$ for $\% 20$ estimating $\%$ 20conditional\%20failure\%20probabilities.pdf.

[2] D. A. Alvarez. On the calculation of the bounds of probability of events using infinite random sets. International journal of approximate reasoning, 43(3):241-267, 2006.

[3] D. A. Alvarez, J. E. Hurtado, and J. Ramírez. Tighter bounds on the probability of failure than those provided by random set theory. Computers \&5 Structures, 189:101-113, 2017.

[4] M. Campi and S. Garatti. Wait-and-judge scenario optimization. Mathematical Programming, pages 1-35, 2016.

[5] M. C. Campi, G. Calafiore, and S. Garatti. New results on the identification of interval predictor models. In Proceedings of the 16th IFAC World Congress, Prague, 2005.

[6] M. C. Campi, S. Garatti, and M. Prandini. The scenario approach for systems and control design. Annual Reviews in Control, 33(2):149-157, 2009.

[7] L. G. Crespo, S. P. Kenny, and D. P. Giesy. Interval predictor models with a linear parameter dependency. Journal of Verification, Validation and Uncertainty Quantification, 1(1), 2016.

[8] M. De Angelis. Efficient Random Set uncertainty quantification by means of advanced sampling techniques. PhD thesis, University of Liverpool, 2015. URL https://core.ac.uk/download/pdf/80774362.pdf.

[9] M. de Angelis, E. Patelli, and M. Beer. Advanced line sampling for efficient robust reliability analysis. Structural safety, 52:170-182, 2015.

[10] B. Echard, N. Gayton, and M. Lemaire. Ak-mcs: an active learning reliability method combining kriging and monte carlo simulation. Structural Safety, 33(2):145-154, 2011.

[11] M. Faes, J. Sadeghi, M. Broggi, M. de Angelis, E. Patelli, M. Beer, and D. Moens. On the robust estimation of small failure probabilities for strong non-linear models. In ASCE-ASME Journal of Risk and Uncertainty in Engineering Systems Part B: Mechanical Engineering, 2019. doi: 10.1115/ 1.4044044 . 
This manuscript version is made available under the CC-BY-NC-ND 4.0

license http://creativecommons.org/licenses/by-nc-nd/4.0/

○ 2019 .

[12] S. Ferson, V. Kreinovich, L. Ginzburg, D. S. Myers, and K. Sentz. Constructing probability boxes and dempster-shafer structures. Technical report, Technical report, Sandia National Laboratories, 2003.

[13] T. Fetz. Efficient computation of upper probabilities of failure. In D. M. F. Christian Bucher, Bruce R. Ellingwood, editor, Safety, Reliability, Risk, Resilience and Sustainability of Structures and Infrastructure, pages 493502. 12th International Conference on Structural Safety \& Reliability, TUVerlag Vienna, 2017.

[14] B. J. Goodno and J. M. Gere. Mechanics of Materials. Cengage Learning, 2016.

[15] X. Huang, J. Chen, and H. Zhu. Assessing small failure probabilities by ak-ss: an active learning method combining kriging and subset simulation. Structural Safety, 59:86-95, 2016.

[16] J. E. Hurtado. Assessment of reliability intervals under input distributions with uncertain parameters. Probabilistic Engineering Mechanics, 32:80-92, 2013.

[17] D. Kececioglu. Robust engineering design-by-reliability with emphasis on mechanical components 85 structural reliability, volume 1. DEStech Publications, Inc, 2003.

[18] H. Panayirci. Efficient solution for galerkin-based polynomial chaos expansion systems. Advances in Engineering Software, 41(12):1277-1286, 2010.

[19] E. Patelli. Handbook of Uncertainty Quantification, chapter COSSAN: A Multidisciplinary Software Suite for Uncertainty Quantification and Risk Management, pages 1-69. Springer International Publishing, Cham, 2016. ISBN 978-3-319-11259-6. doi: 10.1007/978-3-319-11259-6_59-1.

[20] E. Patelli, H. M. Panayirci, M. Broggi, B. Goller, P. Beaurepaire, H. J. Pradlwarter, and G. I. Schuëller. General purpose software for efficient uncertainty management of large finite element models. Finite elements in analysis and design, 51:31-48, 2012.

[21] E. Patelli, D. A. Alvarez, M. Broggi, and M. d. Angelis. Uncertainty management in multidisciplinary design of critical safety systems. Journal of Aerospace Information Systems, 12(1):140-169, 2014. doi: 10.2514/ 1.I010273.

[22] N. K. Prinja, A. Ogunbadejo, J. Sadeghi, and E. Patelli. Structural reliability of pre-stressed concrete containments. Nuclear Engineering and Design, 323:235-244, 2016.

[23] R. Rocchetta and E. Patelli. Stochastic analysis and reliability-cost optimization of distributed generators and air source heat pumps. In System Reliability and Safety (ICSRS), 2017 2nd International Conference on, pages 31-35. IEEE, 2017. 
This manuscript version is made available under the CC-BY-NC-ND 4.0

license http://creativecommons.org/licenses/by-nc-nd/4.0/

(1)2019.

[24] J. Sadeghi, M. de Angelis, and E. Patelli. Robust propagation of probability boxes by interval predictor models. Florianopolis, SC, Brazil, 2018. Proceedings of the joint ICVRAM ISUMA UNCERTAINTIES conference. URL http://icvramisuma2018.org/cd/web/PDF/ ICVRAMISUMA2018-0002.PDF

[25] J. Sadeghi, M. de Angelis, and E. Patelli. Frequentist history matching with interval predictor models. Applied Mathematical Modelling, 61:29-48, 2018.

[26] R. Schöbi and B. Sudret. Propagation of uncertainties modelled by parametric p-boxes using sparse polynomial chaos expansions. In 12th Int. Conf. on Applications of Statistics and Probability in Civil Engineering (ICASP12), 2015.

[27] R. Schöbi and B. Sudret. Structural reliability analysis for p-boxes using multi-level meta-models. Probabilistic Engineering Mechanics, 48:27-38, 2017.

[28] G. I. Schuëller and R. Stix. A critical appraisal of methods to determine failure probabilities. Structural Safety, 4(4):293-309, 1987.

[29] M. Troffaes, T. Fetz, and M. Oberguggenberger. Iterative importance sampling for estimating expectation bounds under partial probability specifications. In The 8th International Workshop on Reliable Engineering Computing (REC2018), Liverpool, UK, pages 1-12, 2018. URL http://rec2018.uk/papers/REC2018-04_M.\%20Troffaes,\%20T. $\% 20 \mathrm{Fetz}, \% 20 \mathrm{M} . \% 200$ berguggenberger_Iterative $\% 20$ Importance $\%$ 20Sampling $\% 20$ or $\% 20$ Estimating $\% 20$ Expectation $\% 20$ Bounds $\% 20$ Under $\%$ 20Partial\%20Probability\%20Specifications.pdf

[30] M. C. Troffaes. Imprecise monte carlo simulation and iterative importance sampling for the estimation of lower previsions. International Journal of Approximate Reasoning, 101:31-48, 2018.

[31] S. S. Wilks. Determination of sample sizes for setting tolerance limits. The Annals of Mathematical Statistics, 12(1):91-96, 1941.

[32] X. Yang, Y. Liu, Y. Zhang, and Z. Yue. Hybrid reliability analysis with both random and probability-box variables. Acta Mechanica, 226(5):1341, 2015 . 